\title{
O CONTROLE EM CASCATA DE SISTEMAS PNEUMÁTICOS DE POSICIONAMENTO
}

\author{
Raul Guenther*
}

\author{
Eduardo André Perondi ${ }^{\dagger}$
}

\author{
*Laboratório de Robótica - CTC - UFSC - Caixa Postal 476,CEP 88040-900 - Florianópolis SC \\ †Departamento de Engenharia Mecânica - Escola de Engenharia - UFRGS, CEP 90050 - 000 - Porto Alegre RS
}

\begin{abstract}
This work deals with the control of pneumatic positioning systems and propose a cascade strategy that alow us to overcome several obstacles to the trajectory tracking in pneumatic systems. The exponential convergence of the closed loop trajectory tracking errors in the ideal case is demonstrated using Lyapunov's method. The robustness properties of the closed loop system are also presented. An experimental implementation attests the effectiveness of this strategy.
\end{abstract}

KEYWORDS: Pneumatic drives, cascade control, robotics, feedback linearizations.

\section{RESUMO}

Este trabalho trata do controle dos sistemas pneumáticos de posicionamento e propõe uma estratégia em cascata que possibilita superação de diversos obstáculos inerentes ao seguimento preciso de trajetórias com estes sistemas. A convergência exponencial dos erros de seguimento em malha fechada no caso ideal é demonstrada utilizando o método de Lyapunov. Apresentam-se também as propriedades de robustez da malha fechada. Uma implementação experimental atesta a eficácia da estratégia.

\footnotetext{
Artigo submetido em $10 / 06 / 02$

1a. Revisão em 06/12/02; 2a. Revisão em 22/04/03

Aceito sob recomendação do Ed. Assoc. Prof. Paulo Eigi Miyagi
}

PALAVRAS-CHAVE: Atuadores pneumáticos, controle em cascata, robótica, linearização por realimentação.

\section{INTRODUÇÃO}

Os sistemas que possibilitam posicionar uma carga mecânica localizada através de uma coordenada " $y$ " são chamados de sistemas de posicionamento. Esta localização pode ser fixa ou variável no tempo, ou seja, $y(t)$, e nestes casos os sistemas de posicionamento são, algumas vezes, chamados de seguidores.

Os sistemas de posicionamento são utilizados em diversas aplicações dentre as quais pode-se citar laminadores, turbinas hidráulicas, máquinas agrícolas, lemes de aeronaves, antenas, telescópios, direcionadores de foguetes, máquinas de precisão e robôs manipuladores.

Um componente importante do sistema é o elemento que aplica a força sobre a carga mecânica para levá-la até a posição desejada. Este elemento é chamado de motor ou acionamento e pode ser elétrico, hidráulico ou pneumático. Uma discussão sobre as características e aplicações de cada um desses motores pode ser encontrada em Bollmann e Guenther (1997). Aqui, discutem-se as características dos sistemas com acionamento pneumático, doravante denominados sistemas pneumáticos de posicionamento, compostos basicamente por uma servoválvula que controla a vazão de ar na direção de um cilindro, a cujo êmbolo está ligada a massa a ser posicionada. 
Os sistemas pneumáticos de posicionamento são relativamente baratos, leves e de fácil instalação, apresentando também uma boa relação entre a força aplicada e o seu peso. Isso os torna bastante atraentes para diversas aplicações.

A estrutura dinâmica do sistema com parcelas oscilatórias introduzidas pela compressibilidade do ar, as não linearidades associadas ao escoamento do ar na servoválvula e ao atrito entre o êmbolo e a camisa do cilindro em conjunção com os atrasos devidos à baixa velocidade de propagação das ondas de pressão no ar, tornam os sistemas pneumáticos de posicionamento difíceis de serem controlados de forma a obter-se o desempenho necessário para diversas tarefas de posicionamento.

O objetivo de desenvolver sistemas pneumáticos de posicionamento rápidos e precisos tem motivado diversos trabalhos em controle (veja seção 4). Busca-se superar os problemas inerentes aos sistemas pneumáticos empregando técnicas de controle adequadas.

Este trabalho é desenvolvido dentro desta linha. Propõe-se aqui uma estratégia de controle em cascata que permite (i) superar as não linearidades associadas ao escoamento do ar na servoválvula, (ii) mantém limitadas as oscilações devido à compressibilidade do ar e, (iii) cria um caminho para enfrentar as dificuldades introduzidas pelo atrito entre o êmbolo e a camisa do cilindro.

A estratégia consiste em interpretar o sistema de posicionamento como dois subsistemas interconectados: um subsistema mecânico comandado por uma lei de controle capaz de garantir sua estabilidade na presença de perturbações, e um subsistema pneumático onde, após uma linearização por realimentação, é empregada uma lei de controle proporcional.

Após a descrição do sistema na seção 2, apresenta-se, na seção 3, sua modelagem dinâmica, utilizada no projeto de controlador e na discussão das características de controle dos sistemas de posicionamento pneumáticos apresentada na seção 4. Nas seções 5, 6 e 7 descreve-se o controlador em cascata e apresentam-se suas propriedades de estabilidade e de robustez. Os resultados experimentais são apresentados e comentados na seção 8 . Na seção 9 delineiam-se algumas conclusões.

\section{O SISTEMA DE POSICIONAMENTO}

Os elementos básicos do sistema em estudo estão esquematizados na Fig. 1. O sistema consiste de uma servoválvula direcional e de um cilindro de dupla ação

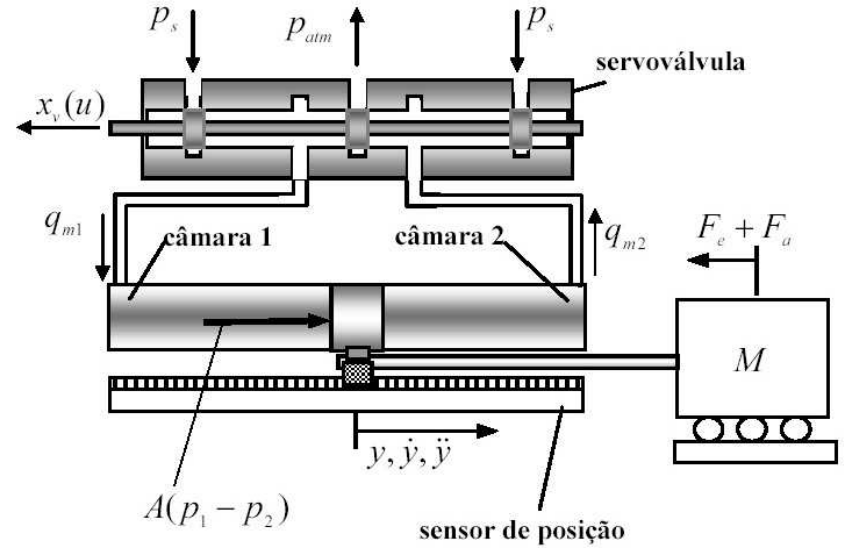

Figura 1: Sistema pneumático de posicionamento.

com transmissão de movimento por fita, cujo êmbolo é conectado a uma carga inercial $(M)$.

A carga inercial é deslocada utilizando a energia pneumática armazenada em um vaso de pressão, onde o ar é mantido a uma pressão de suprimento $p_{s}$. O fluxo de ar sob pressão é direcionado através da servoválvula. Deslocando o carretel da servoválvula de $x_{v}$ para a esquerda, por exemplo, a câmara 1 é conectada com o reservatório e a câmara 2 é conectada com a atmosfera. Como a pressão no reservatório $\left(p_{s}\right)$ é maior do que a pressão na câmara $1\left(p_{1}\right)$, surge uma vazão mássica de ar do reservatório para a câmara $1\left(q_{m 1}\right)$. Simultaneamente, como a pressão na câmara $2\left(p_{2}\right)$ é maior do que a pressão atmosférica $\left(p_{a t m}\right)$, surge uma vazão mássica de ar da câmara 2 para a atmosfera $\left(q_{m 2}\right)$. Com isso a pressão na câmara 1 aumenta e a pressão na câmara 2 diminui. A diferença de pressões $\left(p_{\Delta}=p_{1}-p_{2}\right)$ gera a força que provoca o deslocamento da carga.

Muitas vezes o deslocamento do carretel da válvula $\left(x_{v}\right)$ é provocado por uma tensão $(u)$ aplicada em um solenóide. Desta forma, a dinâmica deste sistema tem como entrada a tensão $u$ e como saída o deslocamento $y$ do êmbolo do cilindro (ou da massa Macoplada). Essa dinâmica compreende a relação entre a tensão aplicada $u$ (entrada) e o deslocamento $x_{v}$ do carretel (dinâmica da válvula), as relações entre as aberturas na válvula (ocasionadas pelo deslocamento do carretel) e as vazões mássicas de ar através da válvula, as relações entre as vazões mássicas nas câmaras do cilindro e as pressões que elas provocam, e a relação entre a diferença de pressões e o deslocamento do êmbolo $y$ (saída). 


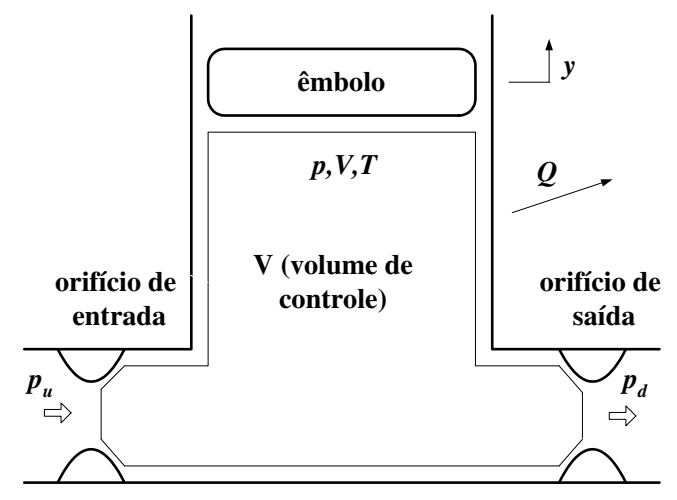

Figura 2: Volume de controle no sistema pneumático.

\section{O MODELO DINÂMICO}

De acordo com o funcionamento do sistema pneumático descrito na seção anterior, o modelo dinâmico estabelece a relação entre a tensão no solenóide $u$ e o deslocamento do êmbolo do cilindroy.

Neste trabalho a dinâmica da válvula (relação entre a tensão aplicada $u$ e o deslocamento do carretel) é considerada suficientemente rápida para poder ser desprezada.

O modelo para o sistema é obtido a partir da descrição das relações entre as vazões mássicas nas câmaras do cilindro e as pressões ocasionadas, e da relação entre a diferença de pressões e o deslocamento do êmbolo $y$. A descrição das vazões mássicas de ar no cilindro é feita usando a conservação da energia. A relação entre a diferença de pressões e o deslocamento do êmbolo é obtida usando a $2^{a}$ lei de Newton.

Considerando o volume de controle indicado na Fig. 2, a conservação da energia é empregada para realizar o balanço entre a energia interna da massa que entra no volume de controle, a potência do movimento do pistão e a variação da energia interna no volume de controle. A pressão a montante (upstream) do orifício é denominada $p_{u}$ e a pressão a jusante (downstream) $p_{d}$.

Para o volume de controle correspondente à câmara 1 , a energia interna da massa que entra é $C_{p} q_{m 1} T$, onde $C_{p}$ é o calor específico do ar a pressão constante, $T$ é a temperatura do ar de suprimento, $q_{m 1}=\left(d m_{1} / d t\right)$ é a vazão mássica na câmara 1 . A potência do movimento do pistão é $p_{1} \dot{V}_{1}$, onde $p_{1}$ é a pressão na câmara $1 \mathrm{e}$ $\dot{V}_{1}=\left(d V_{1} / d t\right)$ é a vazão volumétrica. A variação na energia interna do volume de controle é $d\left(C_{V} \rho_{1} V_{1} T\right) / d t$, onde $C_{V}$ é o calor específico do ar a volume constante e $\rho_{1}$ é a massa específica do ar. Como em Virvalo (1995),
Bobrow e McDonell (1998), Göttert e Neumann (1999) e Brun et al. (2000) a temperatura do ar nas câmaras é assumida como constante e igual à temperatura do ar de suprimento. Definindo a relação de calores específicos $r=C_{P} / C_{V}$ e lembrando que para um gás ideal a equação da massa específica é $\rho_{1}=p /(R T)$, sendo $R$ a constante do gás, pode-se realizar o balanço energético, do qual resulta

$$
q_{m 1} T-\frac{p_{1}}{C_{P}} \frac{d V_{1}}{d t}=\frac{1}{r R} \frac{d}{d t}\left(p_{1} V_{1}\right)
$$

onde a potência calorífica dissipada através das paredes do cilindro $(\dot{Q})$ é desprezada. O volume total na câmara 1 é dado por $V_{1}=A y+V_{10}$, onde $A$ é a área do embolo, $y$ é o deslocamento do embolo e $V_{10}$ é o volume morto na câmara 1 incluindo as tubulações. A taxa de variação deste volume é $\dot{V}_{1}=A \dot{y}$, onde $\dot{y}=d y / d t$ é a velocidade do êmbolo. Com isso, resolvendo a Eq.(1) para $\dot{p}_{1}$, após efetuar a derivada do termo da direita, chega-se a

$$
\dot{p}_{1}=-\frac{A r \dot{y}}{A y+V_{10}} p_{1}+\frac{R r T}{A y+V_{10}} q_{m 1}
$$

onde foi utilizada a relação $C_{p}=(r R) /(r-1)$ (veja por exemplo Streeter, 1981). De forma semelhante, sendo $L$ o comprimento útil do cilindro, para a câmara 2 obtémse:

$$
\dot{p}_{2}=\frac{A r \dot{y}}{A(L-y)+V_{20}} p_{2}+\frac{R r T}{A(L-y)+V_{20}} q_{m 2}
$$

Assumindo que as vazões mássicas são funções das pressões no cilindro e da tensão aplicada à servoválvula $u$, ou seja, $q_{m 1}=q_{m 1}\left(p_{1}, u\right)$ e $q_{m 2}=q_{m 2}\left(p_{2}, u\right)$, as expressões (2) e (3) resultam:

$$
\begin{aligned}
& \dot{p}_{1}=-\frac{A r \dot{y}}{A y+V_{10}} p_{1}+\frac{R r T}{A y+V_{10}} q_{m 1}\left(p_{1}, u\right) \\
& \dot{p}_{2}=\frac{A r \dot{y}}{A(L-y)+V_{20}} p_{2}+\frac{R r T}{A(L-y)+V_{20}} q_{m 2}\left(p_{2}, u\right)
\end{aligned}
$$

O equilíbrio de forças no êmbolo é obtido pela aplicação da $2^{a}$ lei de Newton, e resulta:

$$
M \ddot{y}+F_{a}+F_{e}=A p_{\Delta}
$$

onde $M$ é a massa deslocada, $F_{a}$ é a força de atrito, $F_{e}$ é a força externa e $A p_{\Delta}$ é a força resultante da diferença de pressão entre as câmaras (ver Fig. 3).

As equações (4), (5) e (6) constituem um modelo dinâmico de quarta ordem do sistema pneumático de posicionamento, cujas variáveis de estado podem ser 


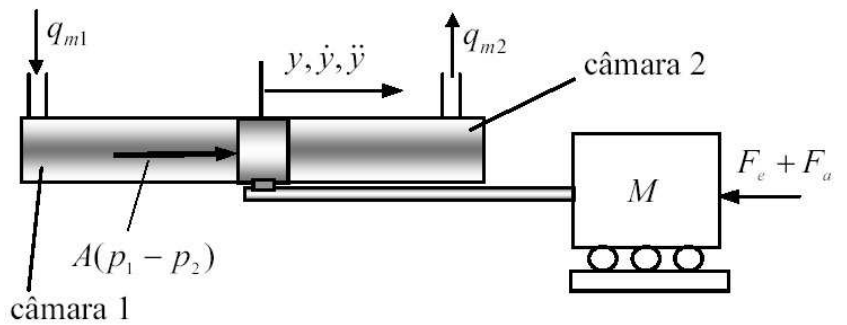

Figura 3: Equilíbrio de forças no êmbolo do cilindro atuador.

$x=\left[\begin{array}{cccc}y & \dot{y} & p_{1} & p_{2}\end{array}\right]^{T}$. Sua não linearidade é caracterizada pelo produto e quociente de variáveis de estado presentes nas relações (4) e (5), pela não linearidade das funções que descrevem as vazões mássicas $q_{m 1}=q_{m 1}\left(p_{1}, u\right)$ e $q_{m 2}=q_{m 2}\left(p_{2}, u\right)$, e pela parcela de atrito estático presente em $F_{a}$.

Observação 1 (modelo linear): Expandindo as funções que descrevem as vazões mássicas em série de Taylor em torno de um ponto de funcionamento chega-se a um modelo linear de quarta ordem com um zero (Perondi e Guenther, 1999). Se o ponto de funcionamento é o ponto central do cilindro o zero cancela um pólo e a função de transferência do sistema de terceira ordem resultante é:

$$
G(s)=\frac{b_{0}}{s\left(s^{2}+a_{2} s+a_{1}\right)}
$$

Observação 2: Utilizando as expressões (4) e (5) podese escrever a derivada da diferença de pressão em relação ao tempo como

$$
\dot{p}_{\Delta}=\hat{h}\left(p_{1}, p_{2}, y, \dot{y}\right)+\hat{u}\left(p_{1}, p_{2}, y, u\right)
$$

onde os termos que dependem da tensão na servoválvula $u$ estão agrupados em uma função $\hat{u}=\hat{u}\left(p_{1}, p_{2}, y, u\right)$, e os termos que independem da tensão estão agrupados em uma função $\hat{h}=\hat{h}\left(p_{1}, p_{2}, y, \dot{y}\right)$, ou seja:

$$
\begin{aligned}
& \hat{u}\left(p_{1}, p_{2}, y, u\right)=\operatorname{Rr} T\left[\frac{q_{m 1}\left(p_{1}, u\right)}{A y+V_{10}}-\frac{q_{m 2}\left(p_{2}, u\right)}{A(L-y)+V_{20}}\right] \\
& \hat{h}\left(p_{1}, p_{2}, y, \dot{y}\right)=-r A \dot{y}\left[\frac{p_{1}}{A y+V_{10}}+\frac{p_{2}}{A(L-y)+V_{20}}\right]
\end{aligned}
$$

As expressões (6) e (8) são uma outra forma de descrever o sistema não linear de quarta ordem e são utilizadas na seção 5 .

\section{O CONTROLE DO SISTEMA}

O objetivo de controle é obter um sistema em malha fechada com uma larga banda de passagem e pequenos erros de seguimento e posicionamento.

As não linearidades introduzidas pelo escoamento do ar e pela força de atrito são dificuldades básicas para atingir este objetivo de controle. Outras podem ser visualizadas a partir do modelo linear apresentado na seção anterior.

O modelo linear tem dois pólos que, com uma realimentação proporcional, são oscilatórios com um amortecimento que diminui na medida em que o ganho proporcional aumenta, e que se dirigem para o semiplano complexo direito a medida que o ganho de realimentação cresce.

Além disso, os parâmetros do modelo linear de terceira ordem $\left(b_{0}, a_{1}\right.$ e $\left.a_{2}\right)$ dependem de características físicas do sistema sujeitas a incertezas. Um exemplo é a massa específica do ar que depende da temperatura ambiente e da pressão de suprimento. Outro é a força de atrito, função das condições de lubrificação. Isto provoca incertezas nos parâmetros do modelo e, consequentemente, incertezas na localização dos pólos. Os parâmetros do modelo linear de quarta ordem dependem também da posição em torno da qual a linearização está sendo realizada. Com o cilindro na posição central (usada para obter o modelo de terceira ordem) o sistema tem a menor freqüência natural, que aumenta a medida que o cilindro é deslocado para as extremidades (Virvalo, 1995).

Visto a partir do modelo linear trata-se, portanto, de um sistema com pólos que se deslocam para a instabilidade com uma realimentação proporcional, cujos parâmetros variam com a posição do êmbolo, e que contém incertezas.

\subsection{Os controladores lineares}

As oscilações pouco amortecidas e o risco de instabilidade detectadas no modelo linear limitam o ganho de um controlador proporcional $(\mathrm{P})$ a pequenos valores. Por isso, realimentado proporcionalmente, o sistema resulta lento em malha fechada. Os pequenos ganhos proporcionais também resultam em grandes erros provocados pelo atrito estático entre o êmbolo e o cilindro (Virvalo, 1995).

Utilizando a técnica do lugar das raízes verifica-se que a introdução de uma parcela derivativa na realimentação (controlador PD) não altera significativamente as dificuldades estruturais discutidas acima (Liu and Bobrow, 1988). 
Uma parcela integral pode auxiliar na redução dos erros de seguimento e posicionamento provocados pelo atrito, mas também não altera a posição dos pólos em malha fechada de forma a reduzir as oscilações pouco amortecidas e os riscos de instabilidade. Além disso, o ajuste dos ganhos dos controladores PID (Proporcional-IntegralDerivativo) depende da trajetória desejada, ou seja, da tarefa (Su and Kuo, 2000). Os controladores PID são insuficientes quando as exigências da tarefa são severas em termos de precisão e velocidade.

Como o modelo linear de terceira ordem (expressão (7)) é controlável, as dificuldades estruturais decorrentes do posicionamento dos pólos em malha aberta podem ser superadas utilizando uma realimentação de estados. Teoricamente, os pólos do sistema em malha fechada podem ser alocados em qualquer posição do plano complexo, o que possibilita obter a resposta especificada através de algum critério de projeto. Na prática o desempenho é limitado pela saturação da vazão na servoválvula (Liu e Bobrow, 1988). Mesmo assim, o controlador com realimentação de estados possibilita a obtenção de velocidades de posicionamento mais elevadas que os controladores PID (Perondi e Guenther, 1999).

Cabe destacar que os controladores de estado são projetados com base no modelo linear. Por isso, os seus ganhos dependem do ponto de funcionamento. Utilizando os mesmos ganhos o desempenho é diferente em cada ponto, podendo, inclusive, ocorrer instabilidades. Essa é outra forte limitação da aplicação dos controladores de estado nos posicionadores pneumáticos.

Nos útimos anos diversos trabalhos tem buscado a superação das limitações dos controladores lineares através da utilização de técnicas de controle não linear.

\subsection{Os controladores não lineares}

O controle a estrutura variável, a linearização por realimentação (veja Slotine and Li, 1991), e o projeto de controladores à partir da interpretação do sistema de posicionamento pneumático como dois subsistemas interconectados, são as técnicas básicas empregadas na última década.

O controle a estrutura variável é uma técnica utilizada para conferir robustez a sistemas com incertezas paramétricas. Sua aplicação em sistemas pneumáticos de posicionamento vem sendo estudada por diversos autores. Thomasset et al. (1993), Surgenor e Vaughan (1995), Perondi e Guenther (2000) utilizaram o modelo linear de terceira ordem (Eq. (7)), considerando as incertezas nos seus parâmetros, para projetar controladores a es-

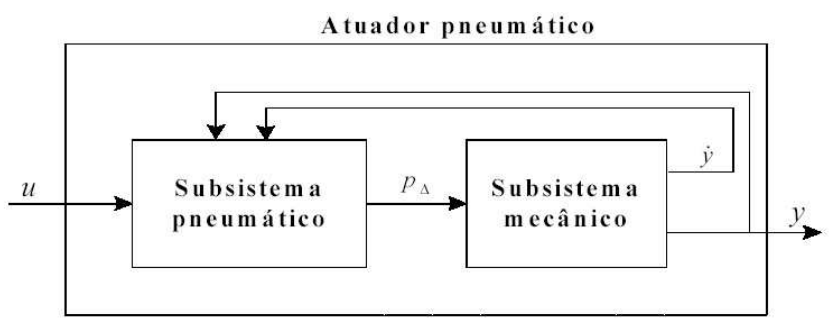

Figura 4: Interpretação do sistema de posicionamento pneumático como dois subsistemas interconectados.

trutura variável, concluindo pela sua superioridade em relação ao controlador de estados no que diz respeito à robustez. A dificuldade de obter, em implementações experimentais, um bom desempenho no seguimento da trajetória sem a ocorrência de oscilações de alta freqüência no sinal de controle, que pode comprometer a vida útil da servoválvula é um desafio a ser superado.

A técnica em que através de uma realimentação de estados adequada busca-se linearizar um sistema é conhecida como linearização por realimentação. A técnica é baseada no modelo do sistema, a partir do qual é feita a linearização. Sua aplicação em sistemas pneumáticos envolve a escolha de um modelo para o comportamento das vazões mássicas em função das pressões e do sinal de controle (veja expressões (4) e (5)). Em Göttert e Neumann (1999) assume-se um modelo em que as vazões mássicas são lineares em relação ao sinal de controle. Mesmo com essa simplificação o seguimento de trajetória resulta melhor do que o obtido com um controlador de estados. Brun et al. (2000) adotam uma representação polinomial para a descrição das vazões mássicas, com resultados qualitativamente semelhantes aos de Göttert e Neumann. Os trabalhos não discutem a robustez em relação à incertezas paramétricas, inerentes aos sistemas pneumáticos, e sabidamente uma deficiência da linearização por realimentação.

A interpretação do sistema pneumático descrito pelas equações (4), (5) e (6) como dois subsistemas interconectados criou outra forma de projetar os controladores. Neste caso considera-se que um subsistema mecânico (6) é acionado por uma força gerada em um subsistema pneumático (4), (5), como na Fig. 4. Projeta-se então a força $A p_{\Delta}$ para que o subsistema mecânico siga a trajetória desejada e, em seguida, a tensão na servoválvula para que o subsistema pneumático forneça as pressões que resultam na força $A p_{\Delta}$.

Essa metodologia de projeto foi empregada por Kawamura et al. (1989) que, utilizando um controlador PI (Proporcional-Integral) no subsistema mecânico e um 
controlador proporcional acrescido de uma parcela para garantir a estabilidade assintótica no subsistema pneumático, mostraram experimentalmente sua eficácia sem, no entanto, apresentar uma comparação de resultados com outros controladores.

A interconecção também foi utilizada por Bobrow e McDonell (1998). Os autores utilizam um controlador semelhante ao proposto em Kawamura et al. (1989), empregando, adicionalmente as inversas das vazões mássicas na servoválvula precisamente levantadas. Apresentam resultados experimentais que mostram um bom desempenho no seguimento de trajetórias e demonstram a estabilidade assintótica da malha fechada, sem discutir o efeito das incertezas no levantamento das inversas das vazões mássicas.

O controlador em cascata proposto na próxima seção utiliza a interpretação descrita na Fig. 4, e possibilita a obtenção de uma malha fechada exponencialmente estável (veja seção 6), em que os efeitos das incertezas dependem dos ganhos (seção7).

\section{O CONTROLE EM CASCATA}

A estratégia em cascata foi desenvolvida a partir de uma metodologia de redução de ordem e foi utilizada com sucesso no controle de acionamentos hidráulicos (Guenther e De Pieri, 1997).

O sistema pneumático é visto como na Fig. 4 e essa interpretação é aplicada ao sistema não linear (6), (8), reescrevendo-o de forma conveniente.

Para tanto define-se o erro no seguimento da diferença de pressão como:

$$
\tilde{p}_{\Delta}=p_{\Delta}-p_{\Delta d}
$$

onde $p_{\Delta d}$ é a diferença de pressão desejada, a ser definida com base na força $g_{d}=A p_{\Delta d}$ que deve ser aplicada à massa para obter um desempenho desejado no posicionamento.

Utilizando a definição (11) as expressões (6) e (8) podem ser reescritas como:

$$
\begin{aligned}
M \ddot{y} & =A p_{\Delta d}+A \tilde{p}_{\Delta}-F_{a}-F_{e} \\
\dot{p}_{\Delta} & =\hat{h}\left(p_{1}, p_{2}, y, \dot{y}\right)+\hat{u}\left(p_{1}, p_{2}, y, u\right)
\end{aligned}
$$

Este sistema está na forma em cascata. A expressão (12) pode ser interpretada como um subsistema mecânico de segunda ordem acionado por uma força desejada $g_{d}=$ $A p_{\Delta d}$ sujeito a uma perturbação na entrada $d=A \tilde{p}_{\Delta}-$
$F_{a}-F_{e}$. A expressão (13) descreve o comportamento do subsistema pneumático de segunda ordem.

A estratégia em cascata consiste em:

(i) Projetar uma lei de controle (força desejada $g_{d}(t)=$ $\left.A p_{\Delta d}(t)\right)$ para o subsistema mecânico (12) de modo que a saída $y(t)$ siga uma trajetória desejada $y_{d}(t)$ na presença da perturbação $d=A \tilde{p}_{\Delta}-F_{a}-F_{e}$; e então

(ii) Projetar uma lei de controle $u(t)$ para que o subsistema (13) gere uma força pneumática $g(t)=$ $A p_{\Delta}(t)$ que siga a força desejada $g_{d}(t)=A p_{\Delta d}(t)$.

Neste trabalho a lei de controle para o subsistema mecânico $g_{d}(t)$ é baseada no controlador proposto em Slotine e Li (1988) e a lei de controle $u(t)$ é sintetizada de forma a obter um bom desempenho no seguimento da força no subsistema pneumático.

\subsection{Seguimento no subsistema mecânico}

De acordo com Slotine e Li (1988) a lei de controle para obter o seguimento de trajetória no subsistema mecânico é dada por

$$
g_{d}=M \ddot{y}_{r}-K_{D} s
$$

onde $K_{D}$ é uma constante positiva, $\dot{y}_{r}$ é uma velocidade de referência e " $s$ " é uma medida do erro de seguimento da velocidade. De fato, $\dot{y}_{r}$ pode ser calculada modificando a velocidade desejada $\dot{y}_{d}$ de acordo com o que segue

$$
\dot{y}_{r}=\dot{y}_{d}-\lambda \tilde{y} ; \tilde{y}=y-y_{d} ; s=\dot{y}-\dot{y}_{r}=\dot{\tilde{y}}+\lambda \tilde{y}
$$

onde $\lambda$ é uma constante positiva.

Substituindo (14) em (12), a dinâmica do erro no subsistema mecânico resulta

$$
M \dot{s}+K_{D} s=A \tilde{p}_{\Delta}-F_{a}-F_{e}
$$

Considere a função não negativa:

$$
2 V_{1}=M s^{2}+P \tilde{y}^{2}
$$

onde $P$ é uma constante positiva definida convenientemente na análise de estabilidade. Utilizando (16), a derivada de (17) em relação ao tempo ao longo das trajetórias do subsistema mecânico resulta:

$$
\dot{V}_{1}=-K_{D} s^{2}+A \tilde{p}_{\Delta} s+P \tilde{y} \dot{\tilde{y}}-\left(F_{a}+F_{e}\right) s
$$

A expressão (18) é usada na análise de estabilidade. 


\subsection{Seguimento no subsistema pneumático}

Para alcançar o seguimento da força no subsistema pneumático propõe-se a lei de controle:

$$
\hat{u}=u_{a}-K_{P} \tilde{p}_{\Delta}-A s
$$

onde $u_{a}$ é um sinal de controle auxiliar, $K_{P}$ é uma constante positiva, $A$ é a área do pistão e $s$ é o erro definido em (16).

Substituindo (19) em (13) obtém-se a dinâmica do subsistema pneumático em malha fechada:

$$
\dot{p}_{\Delta}=\hat{h}\left(p_{1}, p_{2}, y, \dot{y}\right)+u_{a}-K_{p} \tilde{p}_{\Delta}-A s
$$

O projeto do sinal $u_{a}$ e da constante $K_{P}$ é baseado na função escalar não negativa $V_{2}$ :

$$
2 V_{2}=\tilde{p}_{\Delta}^{2}
$$

A derivada de (21) em relação ao tempo é $\dot{V}_{2}=\tilde{p}_{\Delta}\left(\dot{p}_{\Delta}-\right.$ $\dot{p}_{\Delta d}$ ), onde a derivada da diferença de pressão desejada $\left(\dot{p}_{\Delta d}\right)$ é obtida derivando (14). Disso decorre que para calcular $\dot{p}_{\Delta d}$ necessita-se conhecer a aceleração (veja (15)). No caso ideal (parâmetros conhecidos, sem atrito e sem força externa), a aceleração pode ser calculada através da expressão (6) medindo a diferença de pressões $p_{\Delta}$.

Utilizando (20) obtém-se a derivada de (21) ao longo das trajetórias em malha fechada do subsistema pneumático:

$$
\dot{V}_{2}=\tilde{p}_{\Delta}\left[u_{a}+\hat{h}-\dot{p}_{\Delta d}-K_{P} \tilde{p}_{\Delta}-A s\right]
$$

Definindo o sinal de controle auxiliar no caso ideal como

$$
u_{a}^{*}=\dot{p}_{\Delta d}-\hat{h}\left(p_{1}, p_{2}, y, \dot{y}\right)
$$

resulta

$$
\dot{V}_{2}=\tilde{p}_{\Delta}\left[\Delta u_{a}-K_{P} \tilde{p}_{\Delta}-A s\right]
$$

onde $\Delta u_{a}=u_{a}-u_{a}^{*}$ é o erro na estimativa do sinal auxiliar. No caso ideal, $u_{a}=u_{a}^{*}, \Delta u_{a}=0$, e a expressão (24) resulta

$$
\dot{V}_{2}=-K_{P} \tilde{p}_{\Delta}^{2}-A \tilde{p}_{\Delta} s
$$

A expressão (25) é utilizada na análise de estabilidade.

\subsection{Controle do sistema}

O controlador em cascata do sistema de posicionamento é obtido pela combinação da lei (14) para o seguimento no subsistema mecânico com a lei (19) para o subsistema pneumático.

Utilizando (12), (15) e (16) calcula-se a diferença de pressão desejada para obter o seguimento da trajetória no subsistema mecânico, $p_{\Delta d}=g_{d} / A$. Desta forma este subsistema é controlado na linha das forças externas e de atrito, sendo possível estimar e compensar a força de atrito, como é feito em Canudas et al. (1995) para os acionamentos elétricos.

Empregando (19) e (23), no caso em que $u_{a}=u_{a}^{*}$, calcula-se $\hat{u}$. Para tanto necessita-se obter a derivada da diferença de pressão, calculada anteriormente, e a função $\hat{h}=\hat{h}\left(p_{1}, p_{2}, y, \dot{y}\right)$ calculada através de (10).

Calculado o valor de $\hat{u}$, a tensão de controle $u$ é obtida pela inversão da função definida em (9), ou seja, $u=$ $u\left(p_{1}, p_{2}, y, \hat{u}\right)$.

Essa inversão é realizada a partir das funções que descrevem as vazões mássicas $q_{m 1}=q_{m 1}\left(p_{1}, u\right)$ e $q_{m 2}=$ $q_{m 2}\left(p_{2}, u\right)$. As vazões resultam do produto de uma função que depende da tensão na servoválvula $f_{u}(u)$ por uma função das pressões a montante $\left(p_{u}\right)$ e a jusante $\left(p_{d}\right)$ da servoválvula $f_{p}\left(p_{u}, p_{d}\right)$, levantadas experimentalmente de acordo com o procedimento descrito em Perondi e Guenther (2002). Neste trabalho as funções de pressão e de tensão são ajustadas a polinômios de terceira ordem. A inversa $u=u\left(p_{1}, p_{2}, y, \hat{u}\right)$ é obtida calculando as raízes de um polinômio de terceira ordem.

A utilização da função $\hat{h}=\hat{h}\left(p_{1}, p_{2}, y, \dot{y}\right)$ e da inversa $u=u\left(p_{1}, p_{2}, y, \hat{u}\right)$ na lei de controle, pode ser interpretada como uma linearização por realimentação (veja seção 4).

\section{ANÁLISE DE ESTABILIDADE}

Considere o modelo não linear de quarta ordem e o controlador em cascata. Neste caso o sistema em malha fechada é dado por $\Omega=\{(6),(8),(14),(19)\}$. Seja $\rho=\left[\begin{array}{ccc}\tilde{y} & \dot{\tilde{y}} & \tilde{p}_{\Delta}\end{array}\right]^{T}$ o vetor erro de seguimento na malha fechada $\Omega$.

Para o caso ideal, em que os parâmetros do posicionador pneumático são conhecidos e não há força externa $F_{e}$ nem força de atrito $F_{a}$, a convergência exponencial do vetor erro de seguimento $\rho$ é demonstrada no teorema abaixo, utilizando o lema de convergência apresentado a seguir. 
Lema 1 Se a função real $V(t)$ satisfaz a desigualdade $\dot{V}(t)+\alpha V(t) \leq 0$, onde $\alpha$ é um número real, então $V(t) \leq V(0) e^{-\alpha t}$ (veja Slotine e Li, 1991, pp.91).

Teorema 2 Quando os parâmetros do posicionador pneumático são conhecidos e não há força externa $F_{e}$ nem força de atrito $F_{a}$ :

(i) O sistema em malha fechada $\Omega$ é globalmente estável;

(ii) A norma do vetor de erros do sistema $\Omega(\|\rho(t)\|)$ converge exponencialmente para zero.

Prova: A estabilidade global pode ser demonstrada considerando a função não negativa

$$
2 V=2 V_{1}+2 V_{2}=\rho^{T} N_{1} \rho
$$

onde as funções $V_{1}$ e $V_{2}$ foram definidas em (17) e (21), respectivamente. A matriz $N_{1}>0$ é dada por

$$
N_{1}=\left[\begin{array}{lll}
\lambda^{2} M+P & \lambda M & 0 \\
\lambda M & M & 0 \\
0 & 0 & 1
\end{array}\right]
$$

Neste caso (parâmetros conhecidos, sem atrito e sem força externa), $\Delta u_{a}=0 \mathrm{e}$, de acordo com as expressões (18) e (25) a derivada em relação ao tempo de (26) é

$$
\dot{V}=-K_{D} s^{2}+P \tilde{y} \dot{\tilde{y}}-K_{P} \tilde{p}_{\Delta}^{2}
$$

Definindo a constante $P=2 \lambda K_{D}$ e utilizando (15) em (28), resulta $\dot{V}=-\rho^{T} N_{2} \rho$ onde $N_{2}=$ $\operatorname{diag}\left[\begin{array}{ccc}\lambda^{2} K_{D} & K_{D} & K_{p}\end{array}\right]$. A matriz $N_{2}$ é definida positiva e, neste caso,

$$
\dot{V}(t)=-\rho^{T} N_{2} \rho \leq 0
$$

De $(28)$ e $(29)$ conclui-se que $\|\rho(t)\| \rightarrow 0$ para $t \rightarrow \infty$, ou seja, que $y(t) \rightarrow y_{d}(t), \dot{y}(t) \rightarrow \dot{y}_{d}(t)$ e $p_{\Delta}(t) \rightarrow p_{\Delta d}(t)$ para $t \rightarrow \infty$. Portanto, $y(t)$ e $\dot{y}(t)$ são limitados. Com isso, de (14) conclui-se que $p_{\Delta d}(t)$ é limitada. Assim, $p_{\Delta}(t)=p_{1}(t)-p_{2}(t)$ é limitada. Como as pressões $p_{i}(t)$, $\mathrm{i}=1,2$, são limitadas pela pressão de exaustão e pela pressão de suprimento, conclui-se que a norma do estado do sistema é limitada e, portanto, que o sistema em malha fechada $\Omega$ é globalmente estável.

Para mostrar a convergência exponencial do erro, seja $\lambda_{\max }\left(N_{1}\right)$ o maior autovalor da matriz $N_{1}$, e $\lambda_{\min }\left(N_{2}\right)$ o menor autovalor da matriz $N_{2}$, e seja $\gamma=$ $\lambda_{\min }\left(N_{2}\right) / \lambda_{\max }\left(N_{1}\right)$. Como $N_{1}$ e $N_{2}$ são matrizes definidas positivas, estes escalares são todos positivos. Da teoria de matrizes, $N_{1} \leq \lambda_{\max }\left(N_{1}\right) I$ e $\lambda_{\min }\left(N_{2}\right) I \leq N_{2}$, onde $I$ é a matriz identidade. Portanto,

$$
\rho^{T} N_{2} \rho \geq \frac{\lambda_{\min }\left(N_{2}\right)}{\lambda_{\max }\left(N_{1}\right)} \rho^{T}\left[\lambda_{\max }\left(N_{1}\right) I\right] \rho \geq \gamma V(t)
$$

A expressão (30) combinada com (29) permite escrever $\dot{V}(t) \leq-\gamma V(t)$. De acordo com o lema de convergência apresentado acima, este resultado tem como conseqüência:

$$
V(t)=\frac{1}{2} \rho^{T} N_{1} \rho \leq V(0) e^{-\gamma t}
$$

De (31) e usando a relação $\rho^{T} N_{1} \rho \geq \lambda_{\min }\left(N_{1}\right)\|\rho(t)\|^{2}$ pode-se verificar que a norma do vetor erro de seguimento $\rho(t)$ converge exponencialmente para zero com uma velocidade maior que $\gamma / 2$.

Observação: Para o caso ideal (parâmetros conhecidos, sem força externa nem atrito) o controlador em cascata (14), (19) necessita apenas do estado do sistema $x=$ $\left[\begin{array}{llll}y & \dot{y} & p_{1} & p_{2}\end{array}\right]^{T}$ para ser implementado.

\section{PROPRIEDADES DE ROBUSTEZ}

A robustez do sistema em malha fechada $\Omega$ é estabelecida através das propriedades apresentadas abaixo.

Propriedade 1 - Quando os parâmetros são conhecidos e, adicionalmente ao estado, a aceleração é usada na implementação (para calcular $\dot{p}_{\Delta d}$ - veja Eq. (20) e comentário que segue), na presença de uma força externa $F_{e}$ e da força de atrito $F_{a}$, a norma do vetor de erros $\|\rho(t)\|$ converge globalmente para um conjunto residual que depende dos ganhos do controlador.

Isto pode ser verificado lembrando que, neste caso, a derivada em relação ao tempo de (26), de acordo com as expressões (18) e (25) é

$$
\dot{V}(t)=-\rho^{T} N_{2} \rho-\left(F_{a}+F_{e}\right) s
$$

Definindo o vetor força $F$ = $\left[\begin{array}{lll}-\left(F_{a}+F_{e}\right) \lambda & -\left(F_{a}+F_{e}\right) & 0\end{array}\right]^{T}$ e utilizando (14) a expressão (32) pode ser reescrita como:

$$
\dot{V}(t)=-\rho^{T} N_{2} \rho-\rho^{T} F
$$

De (32) observa-se que, mesmo com $N_{2}>0$, a derivada $\dot{V}(t)$ não é definida negativa para todo $\rho \neq 0$.

Utilizando o teorema de Rayleight-Ritz, a partir de (33) pode-se escrever

$$
\dot{V} \leq-\lambda_{\min }\left(N_{2}\right)\|\rho\|^{2}+\|\rho\|\|F\|
$$


e, considerando que existe um limite superior $\bar{F} \geq\|F\|$ para a norma do vetor $F$, ou seja, que a força externa $F_{e}$ e a força de atrito $F_{a}$ são limitadas,

$$
\dot{V} \leq-\lambda_{\min }\left(N_{2}\right)\|\rho\|^{2}+\bar{F}\|\rho\|
$$

De (35) verifica-se que uma condição suficiente para que $\dot{V}(t)$ seja negativa é

$$
\|\rho\|>\frac{\bar{F}}{\lambda_{\min }\left(N_{2}\right)}
$$

Se a condição $(36)$ é satisfeita, $\dot{V}(t)$ é negativa e $V(t)$ decresce. Se a $V(t)$ decresce, então, pela definição (26), a norma do vetor de erros $\|\rho(t)\|$ também decresce. No entanto, se a norma de $\rho$ decresce tal que

$$
\|\rho\| \leq \frac{\bar{F}}{\lambda_{\min }\left(N_{2}\right)}
$$

então $\dot{V}(t)$ pode tornar-se positiva, o que significa que $V(t)$ e conseqüentemente $\|\rho(t)\|$ podem crescer até que a condição (36) seja satisfeita, tornando $\dot{V}(t)$ negativa e fazendo $\|\rho(t)\|$ decrescer.

Isso mostra que $\|\rho(t)\|$ tende para um conjunto residual definido por (37) que depende de $\bar{F}$ e do menor autovalor da matriz $N_{2}$, definido pelos ganhos do controlador $\left(\lambda, K_{D}, K_{P}\right.$ - veja expressão $\left.(29)\right)$.

Propriedade 2 - Quando existe incerteza nos parâmetros, mesmo sem atrito e força externa, o vetor de erros $\rho(t)$ converge localmente para um conjunto residual que depende dos ganhos do controlador.

Esta propriedade pode ser demonstrada considerando que quando os parâmetros têm incertezas o sinal de controle auxiliar é calculado a partir de valores estimados e, ao invés de (23), tem-se

$$
u_{a}=\dot{\bar{p}}_{\Delta d}-\hat{\bar{h}}\left(p_{1}, p_{2}, y, \dot{y}\right)
$$

Neste caso $\Delta u_{a}=u_{a}-u_{a}^{*} \neq 0$ e se a aceleração utilizada para calcular $\dot{\bar{p}}_{\Delta d}$ for obtida a partir de (6) com os parâmetros aproximados, o erro na estimativa do sinal auxiliar $\Delta u_{a}$ é uma função do estado do sistema, ou seja, $\Delta u_{a}=\Delta u_{a}\left(y, \dot{y}, p_{1}, p_{2}\right)$. Disso resulta que a derivada da função não negativa $V_{2}(21)$ ao longo das trajetórias é

$$
\dot{V}_{2}=-K_{P} \tilde{p}_{\Delta}^{2}-A \tilde{p}_{\Delta} s+\tilde{p}_{\Delta} \Delta u_{a}
$$

e, definindo $E(\rho)=\left[\begin{array}{ccc}0 & 0 & \Delta u_{a}\end{array}\right]^{T}$, onde a dependência de $\rho$ decorre do fato do erro $\Delta u_{a}$ ser uma função do estado, a derivada da função $V$ (26) ao longo das trajetórias pode ser escrita como

$$
\dot{V}(t)=-\rho^{T} N_{2} \rho+\rho^{T} E(\rho)
$$

Da mesma forma que na discussão da Propriedade 1 acima, de (40) verifica-se que, mesmo $\operatorname{com} N_{2}>0$, a derivada $\dot{V}(t)$ não é definida negativa para todo $\rho \neq 0$. Novamente utilizando o teorema de Rayleight-Ritz , a partir de (40) pode-se escrever

$$
\dot{V} \leq-\lambda_{\min }\left(N_{2}\right)\|\rho\|^{2}+\|\rho\|\|E(\rho)\|
$$

A partir de uma condição inicial pode-se estabelecer um limite superior para $\|E(\rho)\| \leq \bar{E}$. Assim, $\dot{V} \leq$ $-\lambda_{\min }\left(N_{2}\right)\|\rho\|^{2}+\bar{E}\|\rho\|$.

Pelos mesmos motivos discutidos na propriedade 1 , a norma do vetor de erros $\|\rho(t)\|$ tende para um conjunto residual que depende de $\bar{E}$ e do menor autovalor da matriz $N_{2}$, definido pelos ganhos do controlador $\left(\lambda, K_{D}, K_{P}\right)$, definido por

$$
\|\rho\| \leq \frac{\bar{E}}{\lambda_{\min }\left(N_{2}\right)}
$$

Neste caso, o limite superior $\bar{E}$ é determinado para uma dada condição inicial, o que torna o resultado local.

Propriedade 3 - Quando existe incerteza nos parâmetros na presença de uma força externa $F_{e}$ e da força de atrito $F_{a}$, a norma do vetor de erros $\|\rho(t)\|$ converge localmente para um conjunto residual que depende dos ganhos do controlador.

Este resultado decorre das propriedades 1 e 2 .

\section{RESULTADOS EXPERIMENTAIS}

As propriedades de estabilidade e de robustez apresentadas acima foram comprovadas experimentalmente e os resultados estão apresentados nesta seção.

A bancada experimental ilustrada na Fig. 5 é composta de uma válvula proporcional 5/3 (MPYE-5-1/8 FESTO) que opera um cilindro de dupla ação com transmissão de movimento por fita e diâmetro interno de 0,025 me curso $1 \mathrm{~m}$ (DGPL-1000 FESTO).

O êmbolo móvel do cilindro é conectado a um sensor linear (Festo POT-1000-TFL) de curso igual ao do cilindro atuador $(1,0 \mathrm{~m})$. Três sensores eletroresistivos de pressão medem a pressão absoluta em cada câmara do cilindro e a pressão manométrica de suprimento. 


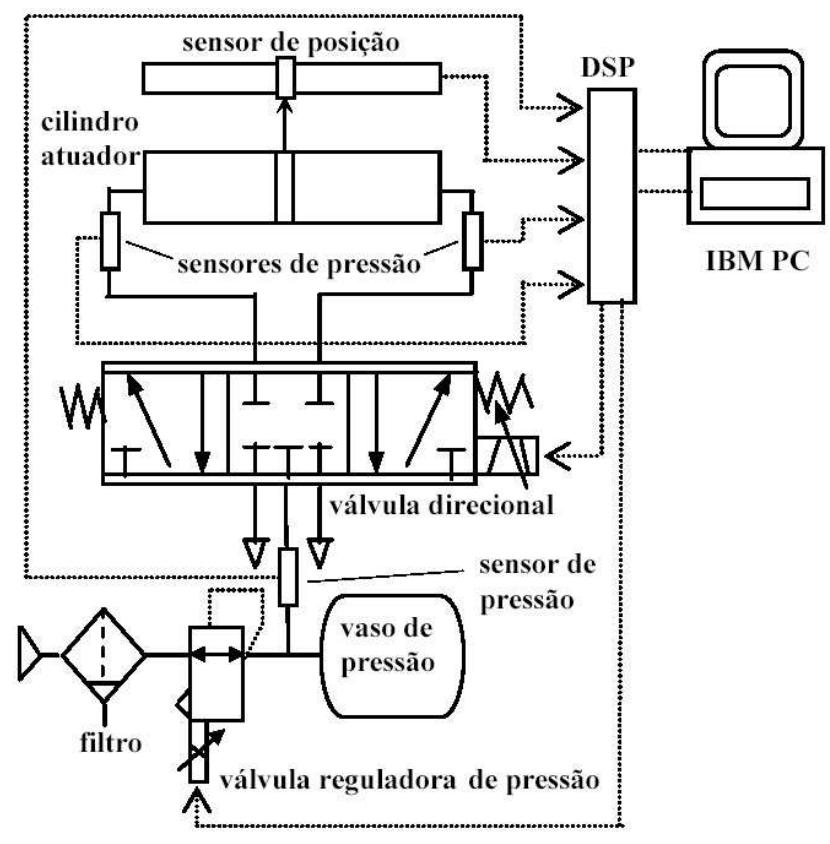

Figura 5: Esquema da bancada experimental.

O aparato de controle e aquisição de dados consiste em uma placa de aquisição de dados rápida (dSPACE GmbH - DS1102) acoplada a um microcomputador IBMPC Pentium $100 \mathrm{MHz}$ com tempo de amostragem de 1 ms. Nas conversões AD e DA, a placa utilizada apresenta programas auxiliares para gerenciamento da aquisição de dados e módulos de acoplamento para o $\mathrm{Ma}$ tLab/Simulink. Assim, os algoritmos em tempo real de medições e controle estão programados via módulo $d S$ PACE para o MatLab/Simulink.

Os parâmetros medidos na bancada são: $A=4,19 \cdot 10^{-4}$ $\mathrm{m}^{2}, r=1,4, R=286,9 \mathrm{~J} \mathrm{Kg} / \mathrm{K}, T=293.15 \mathrm{~K}, L=1$ $\mathrm{m}, V_{10}=1,96 \cdot 10^{-6} \mathrm{~m}^{3}, V_{20}=4,91.10^{-6} \mathrm{~m}^{3}$ e $M=2.9$ $\mathrm{Kg})$.

As funções $q_{m i}\left(p_{i}, u\right), \mathrm{i}=1,2$, em (4) e (5), foram medidas de acordo com o procedimento apresentado em Perondi e Guenther (2001).

Durante a realização dos ensaios não foi aplicada força externa. Portanto, $F_{e}=0$.

Os experimentos foram realizados para uma trajetória desejada senoidal e para uma trajetória desejada polinomial

A trajetória senoidal é $y_{d}(t)=y_{m x} \operatorname{sen}(w t), y_{m x}=$ $0,45 \mathrm{~m}$ e $w=2 \mathrm{rad} / \mathrm{s}$ (veja Fig. 8).

A trajetória desejada polinomial começa com o êmbolo a $0,05 \mathrm{~m}$ da extremidade $\left(y_{d}(0)=0,05 \mathrm{~m}\right)$. A seguir, durante 2 segundos, ocorre um deslocamento de acordo com uma polinomial de sétima ordem até a posição $y_{d}(2)=0,95 \mathrm{~m}$. Esta posição é mantida durante 2 segundos quando é iniciado um movimento segundo a mesma polinomial no sentido contrário, até a posição inicial, onde permanece por 2 segundos (veja Fig. 6). Essa trajetória pode ser descrita por (43) e (44):

$$
\begin{gathered}
y_{d p}(t)=-6 t^{7}+21 t^{6}-25,2 t^{5}+10,5 t^{4} \\
y_{d}(t)[m]= \begin{cases}0,05+y_{d p}(t / 2), & t<2 \\
0,95, & 2 \geq t<4 \\
0,95-y_{d p}((t-4) / 2), & 4 \geq t<6 \\
0,05 & 6 \geq t<8,\end{cases}
\end{gathered}
$$

A freqüência da trajetória senoidal $(w=2 \mathrm{rad} / \mathrm{s})$ e os coeficientes do polinômio de $7^{a}$ ordem foram escolhidos de forma a fornecerem a velocidade máxima recomendada pelo fabricante do cilindro.

Além do controlador em cascata foram utilizados os controladores proporcional $(\mathrm{P})$, proporcional-integralderivativo (PID), e de estados (PVA - posiçãovelocidade-aceleração). Para o controlador $\mathrm{P}$ o sinal de controle é $u=K_{p 1}\left(y-y_{d}\right)$.

Para o PID $u=K_{p 2}\left(y-y_{d}\right)+$ $K_{I 2} \int\left(y-y_{d}\right) d t+K_{v 2}\left(\dot{y}-\dot{y}_{d}\right)$.

Para o controlador de estados a lei de controle é $u=$ $K_{p 3}\left(y-y_{d}\right)+K_{v 3}\left(\dot{y}-\dot{y}_{d}\right)+K_{a 3}\left(\ddot{y}-\ddot{y}_{d}\right)$.

A lei de controle em cascata é dada por (14), (19).

Os ganhos de cada controlador foram sintonizados preliminarmente através de simulações nas quais considerouse que, além da posição e das pressões, a velocidade e a aceleração eram medidas sem ruídos, buscando-se obter o limite para o qual o sinal de controle e a resposta ainda são suaves em todas as trajetórias desejadas (detalhes podem ser encontrados em Perondi, 2002). Em seguida o mesmo procedimento foi realizado na bancada experimental.

Os limites para os ganhos obtidos na bancada experimental foram: controlador P $K_{p 1}=1,3$; PID $K_{p 2}=$ $1,1, K_{I 2}=5,56, K_{v 2}=0,03 ;$ PVA $K_{p 3}=1,3, K_{v 3}=0,03$, $K_{a 3}=0,002$, controlador em cascata $K_{p}=40, K_{D}=40$ e $\lambda=40$.

Estes limites são bastante inferiores aos obtidos em simulações, porque na implementação experimental: (i) a velocidade é obtidas por derivação numérica e, (ii) existe a necessidade de utilizar filtros para reduzir o efeito dos 


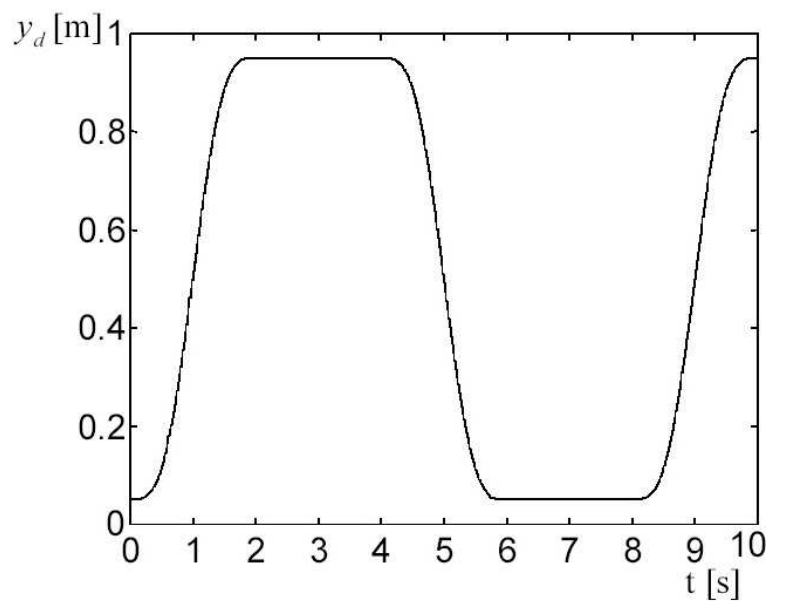

(a) Trajetória desejada.

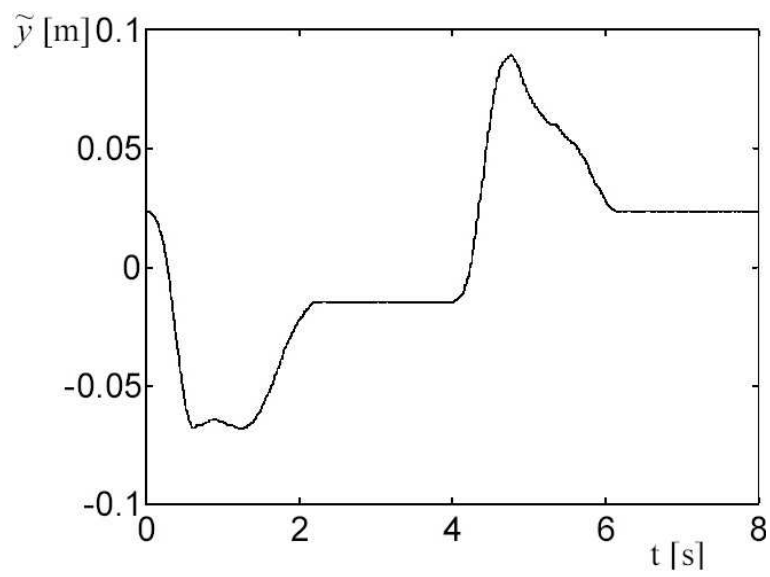

(b) Erro de seguimento.

Figura 6: Trajetória desejada e erro de seguimento para a trajetória polinomial.

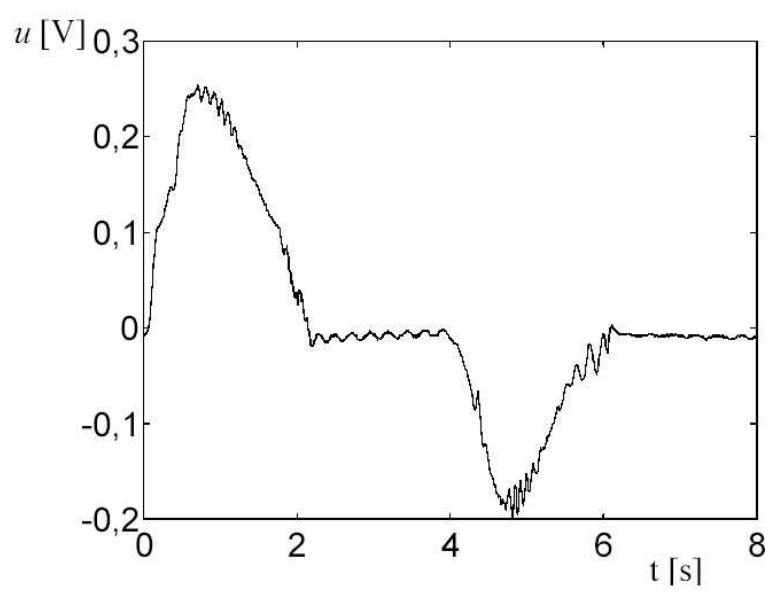

Figura 7: Sinal de controle (trajetória polinomial).

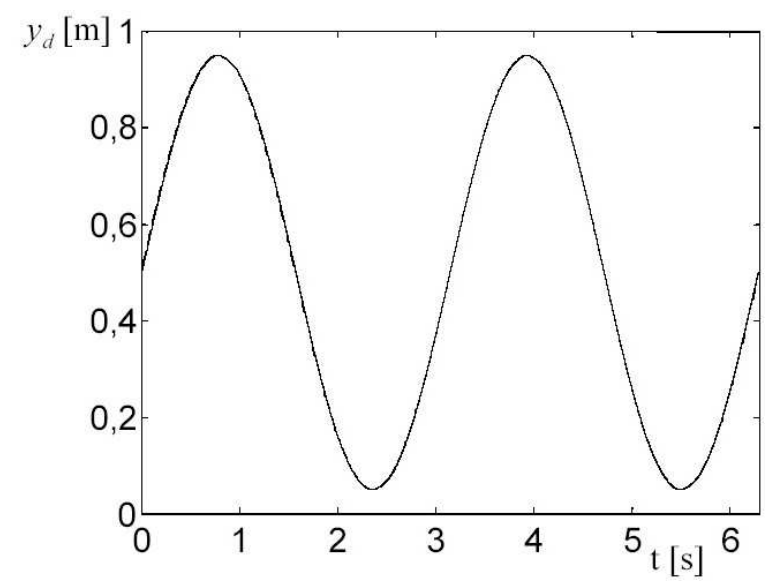

(a) Trajetória desejada.

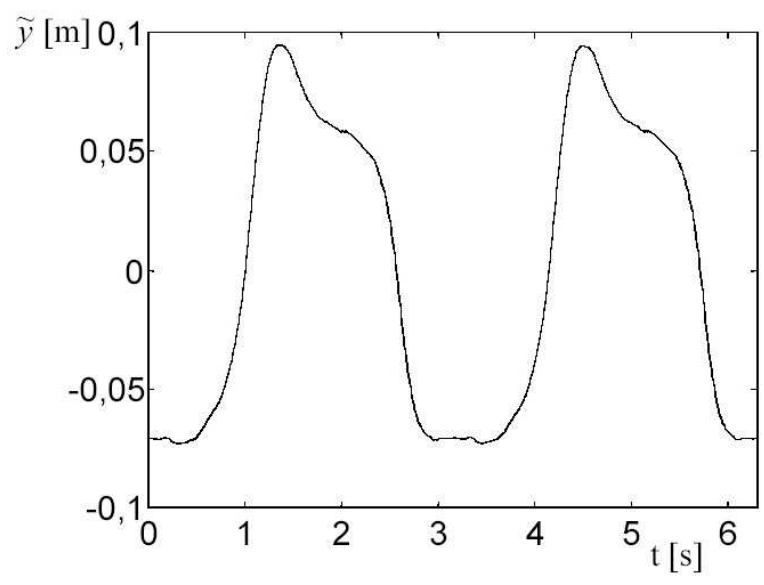

(b) Erro de seguimento.

Figura 8: Trajetória desejada e erro de seguimento para a trajetória senoidal.

ruídos introduzidos pelos sensores. Os filtros digitais Butterworth passa-baixa utilizados tem as seguintes características:

Tabela 1: Característica dos filtros

\begin{tabular}{|c|c|c|}
\hline $\begin{array}{c}\text { Variável } \\
\text { filtrada }\end{array}$ & Ordem & $\begin{array}{c}\text { Freqüência de } \\
\text { corte }[\mathrm{rad} / \mathrm{s}]\end{array}$ \\
\hline Posição & 2 & 350 \\
\hline Velocidade & 1 & 40 \\
\hline Aceleração & 1 & 40 \\
\hline Pressões & 1 & 100 \\
\hline
\end{tabular}

Na Fig. 6 está mostrada a trajetória polinomial desejada e o erro de seguimento obtido com o controlador em cascata. Pode-se observar que o erro converge para um conjunto residual, de acordo com as propriedades de robustez descritas na seção 7. Neste caso o erro residual 


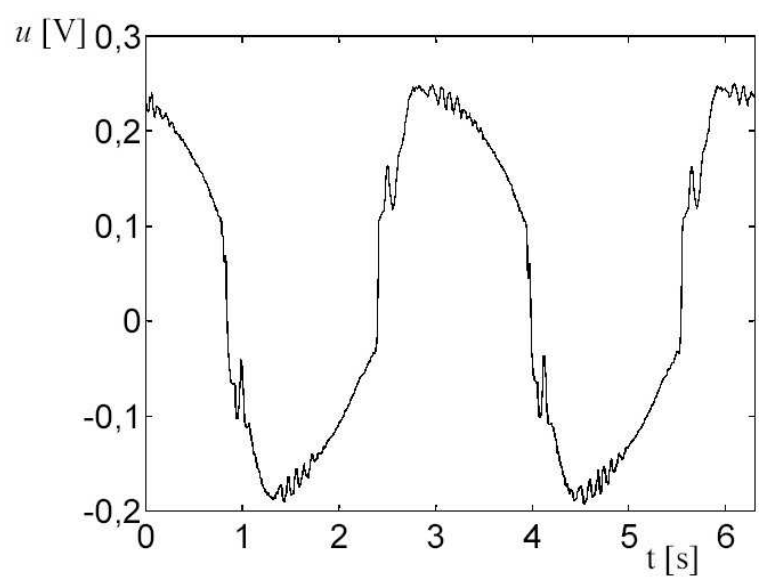

Figura 9: Sinal de controle (trajetória senoidal).

depende não só da força de atrito, mas também das incertezas nos parâmetros inerentes aos dados da bancada. O sinal de controle está mostrado na Fig. 7.

A assimetria no erro de seguimento e no sinal de controle é ocasionada pela assimetria no atrito verificada na identificação da bancada experimental apresentada em Perondi e Guenther (2002). As oscilações de alta frequência no sinal de controle (Fig. 7) são provocadas pelos atrasos introduzidos pelos filtros. Essa afirmação é suportada em simulações realizadas com e sem o uso dos filtros (Perondi, 2002). As oscilações de baixa frequência no sinal de controle durante os períodos de repouso do pistão ocorrem devido ao atrito.

A trajetória senoidal e o respectivo erro de seguimento são mostrados na Fig. 8. O sinal de controle para a trajetória senoidal é apresentado na Fig. 9 podendo-se observar oscilações de alta frequência provocadas pelo emprego dos filtros e oscilações de maior amplitude nos instantes em que o movimento muda de direção, provocadas pelo atrito. As oscilações no sinal de controle estão dentro do limite aceitável para o funcionamento e durabilidade da servoválvula, para as duas trajetórias. Essas oscilações definiram, para ambos os casos, o limite dos ganhos dos controladores e, portanto, do desempenho da malha fechada.

As Figuras 8 e 9 mostram mais uma vez a assimetria no erro e no sinal de controle ocasionadas pela assimetria no atrito. Este conjunto de resultados (Figuras 6 a $9)$ comprova as propriedades do controlador em cascata apresentadas nas seções 6 e 7. Além disso aponta as limitações de desempenhointroduzidas pelo emprego de filtros e pela ação do atrito.

Nas Figuras 10 e 11 são mostrados os erros de segui-

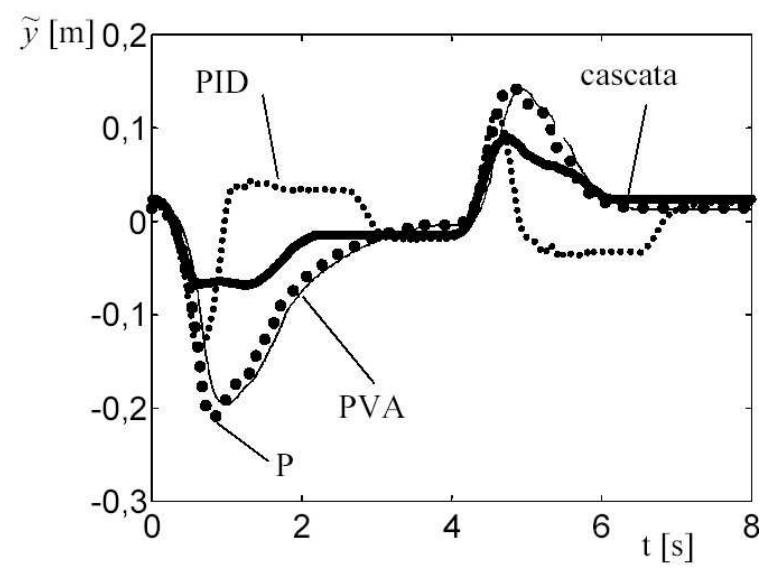

Figura 10: Erros de seguimento (trajetória polinomial) com os controladores P, PID, PVA e em cascata.

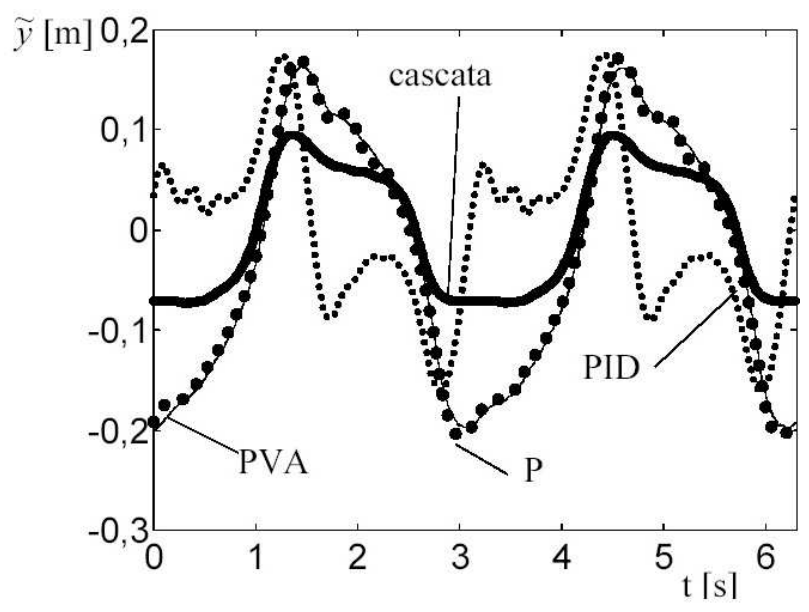

Figura 11: Erros de seguimento para a trajetória senoidal obtidos com os controladores P, PID, PVA e em cascata.

mento para as trajetórias polinomial e senoidal, respectivamente, obtidos utilizando os controladores PID, PVA e em cascata. Estes resultados destacam o desempenho dinâmico alcançado com o controlador em cascata.

\section{CONCLUSÕES}

Neste trabalho é proposto um novo controlador em cascata para sistemas pneumáticos de posicionamento. Demonstra-se a convergência exponencial dos erros de seguimento da malha fechada para o caso em que todos os parâmetros são conhecidos e não existe força externa, nem força de atrito. São estabelecidas as propriedades de robustez da malha fechada. 
As propriedades da malha fechada são comprovadas experimentalmente.

As limitações de desempenho introduzidas pela necessidade do uso de filtros são verificadas experimentalmente.

O desempenho da malha fechada obtido experimentalmente é analisado e comparado ao alcançado utilizando controladores clássicos, comprovando a eficiência do controlador proposto.

A experimentação mostra a importância da compensação do atrito, destacando assim a relevância do caminho que a metodologia de controle em cascata abre neste sentido.

\section{REFERÊNCIAS}

Bobrow, J.E., McDonell,W., (1998). Modeling, Identification, and Control of a Pneumatically Actuated, Force Controllable Robot, IEEE Transactions on Robotics and Automation, vol.15, $\mathrm{n}^{\circ}$ 5, pp. 732-742.

Bollmann, A., Guenther, R., (1997), Posicionadores Hidráulicos e Pneumáticos: Características e Técnicas de Controle. Anais do $5^{\circ}$ Seminário Nacional de Hidr. e Pneum., SENAI/CTAI. Florianópolis. SC, pp 57-78.

Brun,X., Thomasset, D., Bideaux, E., Scavarda, S., (2000), An Accurate Tracking Control of an Electro pneumatic Actuator. $1^{\text {st }}$ FPNI - PhD Symposium, Fluid Power Net International, Hamburgo, pp 215226.

Canudas de Wit, C., Olsson, H., Astrom, K.J. e Lischinsky, P., (1995), A New Model for Control Systems with Friction. IEEE Transactions on Automatic Control, vol. 40, n ${ }^{\circ} 3$, pp.419-425.

Göttert, M., Neumann, R., (1999) Nichlineare Regelungskonzepte für Servopneumatische Roboter. $3^{\text {th }}$ German-Polish seminar in Innovation and Progress in Fluid Engineering, Zakopane, Po.

Guenther, R., De Pieri, E. R., (1997), Cascade Control of the Hydraulic Actuators. Revista Brasileira de Ciências Mecânicas, vol. 19, no 2, Junho, pp. 108120 .

Kawamura, S., Miyata, K., Hanafusa, H., Isida, K., (1989), PI type hierarchical feedback control scheme for pneumatic robots. Proceedings of the 1989 IEEE International Conference on Robotics and Automation. Vol.3, 14-19, Pp. 1853-1858.
Liu, S., Bobrow, J., (1988), An Analysis of a Pneumatic Servo System and its Application to a Computercontrolled Robot. ASME Journal of Dynamic Systems, Measurement and Control, Vol. 110, pp 427433.

Perondi, E.A., Guenther, R., (1999), Controle em Malha Fechada de um Servoposicionador Pneumático. Anais do XV Congr. Bras. de Eng. Mec. Águas de Lindóia, SP.

Perondi, E.A. e Guenther, R., (2000), Controle de um Servoposicionador Pneumático por Modos Deslizantes, Congresso Nacional de Eng. Mecânica - Natal, RN.

Perondi, E.A., e Guenther, R., (2002). Modelagem de um Servoposicionador Pneumático com Atrito. Revista Ciência \& Engenharia (Science and Engineering Journal). UFBA, Uberlândia, MG, Brasil (a ser publicado).

Perondi, E.A. (2002), Controle em Cascata de um Servoposicionador Pneumático com Compensação do Atrito. Florianópolis. Tese de doutorado em Engenharia Mecânica. Centro Tecnológico. UFSC - Flplis. SC.

Slotine e Li (1988), Adaptive Manipulator Control: A Case Study. IEEE Transaction on Automatic Control, Vol. 33, No. 11, pp. 995-1003.

Slotine, J.J.E., Li, W., (1991), Applied Nonlinear Control, Prentice-Hall Inc.

Su, W.-C., Kuo, C.-Y., (2000), Variable structure control of a rodless pneumatic servoactuator with discontinuous sliding surfaces. Proc. ACC, Vol.3, Pp.1617 -1621.

Surgenor, B. W., Vaughan, N. D. and Uebling, M., (1995) Continuous Sliding Mode Control of a Pneumatic Positioning System. Proc. of Eighth Bath International Fluid Power Workshop, pp 270-283.

Thomasset, D., Richard, E., Scavarda, S., Lin, X.F., Sesmat, S., Bouhal, A., (1993), Control of an electro-pneumatic servodrive: a state-affine or a sliding approach. IFAC World Congress, Sydney, pp 501-504.

Virvalo, T., (1995), Modeling and Design of a Pneumatic Position Servo System Realized with Commercial Components. PhD Thesis, Tampere, Finland. 